\title{
ZETA REGULARIZED PRODUCTS
}

\author{
J. R. QUINE, S. H. HEYDARI AND R. Y. SONG
}

\begin{abstract}
If $\lambda_{k}$ is a sequence of nonzero complex numbers, then we define the zeta regularized product of these numbers, $\prod_{k} \lambda_{k}$, to be $\exp \left(-Z^{\prime}(0)\right)$ where $Z(s)=\sum_{k=0}^{\infty} \lambda_{k}^{-s}$. We assume that $Z(s)$ has analytic continuation to a neighborhood of the origin. If $\lambda_{k}$ is the sequence of positive eigenvalues of the Laplacian on a manifold, then the zeta regularized product is known as $\operatorname{det}^{\prime} \Delta$, the determinant of the Laplacian, and $\prod_{k}\left(\lambda_{k}-\lambda\right)$ is known as the functional determinant. The purpose of this paper is to discuss properties of the determinant and functional determinant for general sequences of complex numbers. We discuss asymptotic expansions of the functional determinant as $\lambda \rightarrow-\infty$ and its relationship to the Weierstrass product. We give some applications to the theory of Barnes' multiple gamma functions and elliptic functions. A new proof is given for Kronecker's limit formula and the product expansion for Barnes' double Stirling modular constant.
\end{abstract}

\section{INTRODUCTION}

If $\lambda_{k}$ is a sequence of nonzero complex numbers, then we define the zeta regularized product of these numbers, $\prod_{z k} \lambda_{k}$, to be $\exp \left(-Z^{\prime}(0)\right)$ where $Z(s)=$ $\sum_{k=0}^{\infty} \lambda_{k}^{-s}$. We assume that $Z(s)$ has meromorphic continuation with at most simple poles, to a half-plane containing the origin, and is analytic at the origin. We call such a sequence zeta regularizable. If $\lambda_{k}$ is the sequence of positive eigenvalues of the Laplacian on a manifold, then the zeta regularized product is known as $\operatorname{det}^{\prime} \Delta$, the determinant of the Laplacian. This definition was introduced by Ray and Singer [RS]. A study of some of the extremal properties of the determinant as a function on the space of metrics on surfaces was done in [OPS].

For these sequences of eigenvalues, the functional determinant $\prod_{z k}\left(\lambda_{k}-\lambda\right)$ was discussed by Voros [Vo] and its relationship to the Weierstrass product established. In addition it was shown that by a representation of the functional determinant as a Laplace integral one could obtain an asymptotic expansion for it as $\lambda \rightarrow-\infty$. Voros illustrated his theory of zeta regularized products by several examples and used it to derive formulas for the functional determinant on a closed Riemann surface of genus $g>1$ in terms of the Selberg zeta

Received by the editors October 22, 1990 and, in revised form, April 5, 1991.

1980 Mathematics Subject Classification (1985 Revision). Primary 30D10, 58G25, 33A70, $11 \mathrm{M} 41,33 \mathrm{~A} 25$; Secondary 81E30, 11E45, $11 \mathrm{~F} 72$.

Key words and phrases. Determinant of the Laplacian, zeta function, Barnes' double gamma function, multiple gamma function, zeta regularization, Weierstrass product, Kronecker limit formula. 
function. He also illustrated its application to the theory of Barnes' double gamma function with real zeros.

The sequence of eigenvalues of the Laplacian on a Riemann surface with metric is such that the trace of the heat kernel, $\sum_{k=0}^{\infty} e^{-\lambda_{k} t}$, has a full asymptotic expansion as $t \rightarrow 0^{+}$[MS, G]). This expansion is given in terms of the geometry of the surface. The theory of Voros applies to more general sequences of positive numbers such that the sum above has an asymptotic expansion as $t \rightarrow 0^{+}$.

The purpose of this paper is to extend the theory of Voros to more general sequences of complex numbers. More care is needed in this situation because the product depends on the choice of $\arg \lambda_{k}$. The number of applications of the theory expands, however. The theory of multiple gamma functions with complex zeros and their asymptotic expansions due to Barnes [B1, B2, B3] as well as parts of elliptic function theory may be considered as a special case of the theory of zeta regularized products. It is possible, in addition, to study the determinants the $\partial / \partial z$ and $\partial / \partial \bar{z}$ operator on a genus 1 Riemann surface, operators which have complex eigenvalues. The theory also provides an understanding of the functional equations for simple and multiple gamma functions, the Dedekind eta function, and the Jacobi theta function.

Properties (1) and (2) below follow from the definition of zeta regularized products. In Theorem 1, we show that if $\sum \lambda_{k}^{-s}$ is zeta regularizable, then so is $\sum\left(\lambda_{k}-\lambda\right)^{-k}$. In Theorem 2 , we establish that the relationship given by Voros [Vo] between the zeta regularized and Weierstrass products holds for complex sequences. Although the proof given by Voros can probably be extended to this situation, our proof follows a different approach. Theorems 3 and 4 summarize well-known results about the analytic extensions and asymptotic expansions of zeta functions using a representation as Laplace type integrals of $\sum_{k=0}^{\infty} e^{-\lambda_{k} t}$ together with an asymptotic expansion of the latter as $t \rightarrow 0^{+}$.

Example 7 shows how Barnes' double gamma function is represented as a zeta regularized product associated with the doubly indexed sequence $\lambda_{m n}=$ $m u+n v, m, n \geq 0$. This follows closely Barnes' later approach to multiple gamma functions [B3]. His earlier approach [B1] was to define the double gamma function as a Weierstrass product with an exponential factor, this factor being chosen to give the nicest functional equations (22). The definition as a zeta regularized product gives these functional equations naturally using property (2) of this product. The constants in the exponential factor before the Weierstrass product are given by Theorem 2. Barnes called these modular constants and defined them as constants in certain asymptotic expansions. Their role is analogous to that of the Euler constant for the simple gamma function. Theorem 2 shows they can also be described in terms of the finite parts and residues at integer points of the zeta function of the sequence. We demonstrate below the equivalence of these two descriptions in the case of the double gamma function.

The methods described in Theorems 3 and 4 can be used to derive the asymptotic expansion at infinity of the double gamma function (32). The constant term in this expansion, $\rho_{2}(u, v)$, is called the double Stirling modular constant. Barnes gave an expression for it as a contour integral. Shintani [S1] gave an expression for it as an infinite product (see equations (20) and (21)). His proof depends on results from an earlier paper [S2]. We give a short, self-contained 
derivation of this formula based on the theory developed here.

In Example 9 we show how the ideas used here can be used to give a new proof of Kronecker's limit formula. See [S1] and the references given there for other proofs of it. This formula can be used to give an expression for the determinant of the Laplacian on a flat torus of modulus $\tau$. In Examples 10-13 we derive expressions for the Dedekind eta function and Jacobi theta function as zeta regularized products, and derive an expression for a zeta regularized determinant of $\frac{\partial}{\partial \bar{z}}$ (an operator with complex eigenvalues) on the torus. We note the fact that, contrary to expectation, $\operatorname{det}^{\prime} \frac{\partial}{\partial z} \operatorname{det}^{\prime} \frac{\partial}{\partial \bar{z}} \neq \operatorname{det}^{\prime} \frac{\partial}{\partial z} \frac{\partial}{\partial \bar{z}}$.

For other applications of these techniques, we note papers [QS and CQ]. In [QS] we derive an asymptotic expansion for a generalized factorial which is related to the Kronecker limit formula. In [CQ] we provide factorizations of $\prod_{z}\left(\lambda_{k}-\lambda\right)$ into multiple gamma functions, where $\lambda_{k}$ is the sequence of eigenvalues of the Laplacian or conformal Laplacian on an $n$ sphere.

\section{MAIN THEOREMS}

Suppose $\lambda_{k}$ is a sequence of nonzero complex numbers such that

$$
\alpha=\inf \left\{\left.s \in \mathbf{R}\left|\sum_{k=0}^{\infty}\right| \lambda_{k}\right|^{-s}<\infty\right\}
$$

is positive and finite. The zeta function $\sum_{k=0}^{\infty} \lambda_{k}^{-s}=Z(s)$ is defined and analytic for $\operatorname{Re} s>\alpha$. If the sequence is zeta regularizable, then we define the zeta regularized product

$$
\prod_{k=0}^{\infty} \lambda_{k}
$$

to be $\exp \left(-Z^{\prime}(0)\right)$. We remark that the definition of the zeta regularized product depends on the choices of $\arg \lambda_{k}$, although if a finite number of arguments are changed by an integer multiple of $2 \pi$, the product will not change. In general we will assume the set $\left\{\arg \lambda_{k}\right\}$ is bounded. Since the series for $Z(s)$ converges absolutely, the product is the same for any rearrangement of the sequence.

Two properties of the zeta regularized product are easily established from the definition. First, if $a$ is any nonzero complex number, then

$$
\prod_{k=0}^{\infty} a \lambda_{k}=a^{Z(0)} \prod_{k=0}^{\infty} \lambda_{k}
$$

provided the arguments are chosen so that $\arg a \lambda_{k}=\arg a+\arg \lambda_{k}$. Secondly, if $\lambda_{k}$ is the union of two sequences $\lambda_{k}^{1}$ and $\lambda_{k}^{2}$, renumbered in any way then

$$
\prod_{k=0}^{\infty} \lambda_{k}=\prod_{k=0}^{\infty} \lambda_{k}^{1} \prod_{k=0}^{\infty} \lambda_{k}^{2} .
$$

In principle, the properties are easy to use, but $Z(0)$ must be determined and care must be taken with $\arg \lambda_{k}$. The examples will illustrate this.

For $\lambda \neq \lambda_{k}$ we will also consider the shifted sequences, $\lambda_{k}-\lambda$, and the associated zeta function, $Z(s,-\lambda)=\sum_{k=0}^{\infty}\left(\lambda_{k}-\lambda\right)^{-s}$, which converges for $\operatorname{Re} s>\alpha$. In determining the choice of $\arg \left(\lambda_{k}-\lambda\right)$ from the choice of $\arg \lambda_{k}$, 
we adopt the following convention: for $\left|\lambda_{k}\right|$ large, $\arg \left(\lambda_{k}-\lambda\right)$ is near $\arg \lambda_{k}$. This will define the product unambiguously.

Theorem 1. If $\lambda_{k}$ is zeta regularizable, then so is $\lambda_{k}-\lambda$.

Proof. Let $\lambda$ be fixed. For $\left|\lambda_{k}\right|$ sufficiently large, Taylor expansion gives

$$
\left(\lambda_{k}-\lambda\right)^{-s}=\lambda_{k}^{-s} \sum_{j=0}^{\infty}\left(\begin{array}{c}
s+j-1 \\
j
\end{array}\right)\left(\frac{\lambda}{\lambda_{k}}\right)^{j} \text {. }
$$

Let $h=[\alpha]$ and define

$$
G_{k}(s)=\left(\lambda_{k}-\lambda\right)^{-s}-\sum_{j=0}^{h}\left(\begin{array}{c}
s+j-1 \\
j
\end{array}\right) \lambda_{k}^{-(s+j)} \lambda^{j} .
$$

We have $\left|\left(\begin{array}{c}s+j+h \\ j+h+1\end{array}\right)\right| \leq(|s|+1)^{h+1}\left(\begin{array}{c}|s|+j-1 \\ j\end{array}\right)$ so

$$
\begin{aligned}
\left|G_{k}(s)\right| & =\left|\sum_{j=h+1}^{\infty}\left(\begin{array}{c}
s+j-1 \\
j
\end{array}\right) \lambda_{k}^{-(s+j)} \lambda^{j}\right| \\
& \leq(|s|+1)^{h+1}\left|\lambda_{k}^{-(h+1+s)}\right||\lambda|^{h+1}\left(1-\left|\frac{\lambda}{\lambda_{k}}\right|\right)^{-|s|} .
\end{aligned}
$$

Now for $s$ in a compact set, $\left|\lambda_{k}^{-s}\right|=\left|\lambda_{k}\right|^{-\operatorname{Re} s} \exp \left(\left(\arg \lambda_{k}\right)(\operatorname{Im} s)\right) \leq C_{1}\left|\lambda_{k}\right|^{-\operatorname{Re} s}$ since $\left\{\arg \lambda_{k}\right\}$ is bounded so for $\left|\lambda_{k}\right|$ sufficiently large,

$$
\left|G_{k}(s)\right| \leq C_{2}|\lambda|^{h+1}\left|\lambda_{k}\right|^{-(h+1+\operatorname{Re} s)} \text {. }
$$

Now (4) shows that the function

$$
F(s)=\sum_{k=0}^{\infty} G_{k}(s)
$$

converges absolutely and uniformly on compact subsets of $\operatorname{Re} s>\alpha-(h+1)$ to an analytic function of $s$. This open half-plane contains $s=0$. By (3), for $\operatorname{Re} s>\alpha$ we have

$$
F(s)=\sum_{k=0}^{\infty}\left(\lambda_{k}-\lambda\right)^{-s}-\sum_{j=0}^{h}\left(\begin{array}{c}
s+j-1 \\
j
\end{array}\right) Z(s+j) \lambda^{j}
$$

Equation (6), then, gives us a meromorphic continuation of $\sum_{k=0}^{\infty}\left(\lambda_{k}-\lambda\right)^{-s}$, to a half-plane containing 0 .

We now establish that for a zeta regularizable complex sequence, the relationship between the zeta regularized product and the Weierstrass product given by Voros [Vo] holds. Recall that if $h$ is an integer such that $h+1>\alpha$ we can define the Weierstrass product

$$
W_{h}(\lambda)=\prod_{j=0}^{\infty}\left(1-\frac{\lambda}{\lambda_{j}}\right) \exp \left[P_{h}\left(\frac{\lambda}{\lambda_{j}}\right)\right]
$$

where $P_{h}(x)=x+\frac{x^{2}}{2}+\cdots+\frac{x^{h}}{h}$. At any $s_{0}$, we define the finite part of $Z$ at $s_{0}, F P Z\left(s_{0}\right)$, to be the constant term in the Laurent expansion for $Z$ at $s_{0}$. Also let $\operatorname{Res} Z\left(s_{0}\right)$ denote the residue of $Z$ at $s_{0}$. Note that $\operatorname{Res} Z\left(s_{0}\right)=0$ if $s_{0}$ is not a pole. 
Theorem 2. Suppose $h$ is an integer with $h+1>\alpha$, and that $F$ has poles of order at most 1 at integer points then

$$
\frac{\prod_{z}\left(\lambda_{k}-\lambda\right)}{\prod_{z} \lambda_{k}}=e^{-Q_{h}(\lambda)} W_{h}(\lambda)
$$

where

$$
Q_{h}(\lambda)=\sum_{j=1}^{h} F P Z(j) \frac{\lambda^{j}}{j}+\sum_{j=2}^{h} \operatorname{Res} Z(j)\left(1+\frac{1}{2}+\cdots+\frac{1}{j-1}\right) \frac{\lambda^{j}}{j} .
$$

Proof. From (3) and (5) above we have

$$
F^{\prime}(0)=\sum_{k=0}^{\infty}\left\{-\log \left(\lambda_{k}-\lambda\right)+\log \lambda_{k}-P_{h}\left(\frac{\lambda}{\lambda_{k}}\right)\right\}
$$

hence

$$
W_{h}(\lambda)=\exp \left(-F^{\prime}(0)\right)
$$

Now using (6) above we get

$$
F^{\prime}(0)=Z^{\prime}(0,-\lambda)-Z^{\prime}(0)-\left.\sum_{j=1}^{h} \frac{d}{d s}\left(\begin{array}{c}
-s \\
j
\end{array}\right) Z(s+j)\right|_{s=0}(-\lambda)^{j} .
$$

To evaluate the derivative above, write $Z(s+j)=A_{j} / s+B_{j}+O(s)$ for $s$ near 0 . Then for $j \neq 1$

$$
\left(\begin{array}{c}
-s \\
j
\end{array}\right) Z(s+j)=\frac{A_{j}}{j}(-1)^{j+1}\left(1+s\left(1+\frac{1}{2}+\cdots+\frac{1}{j-1}\right)\right)+(-1)^{j+1} s \frac{B_{j}}{j}+O\left(s^{2}\right) .
$$

Hence

$$
\left.\frac{d}{d s}\left(\begin{array}{c}
-s \\
j
\end{array}\right) Z(s+j)\right|_{s=0}=\frac{(-1)^{j+1}}{j}\left\{A_{j}\left(1+\frac{1}{2}+\cdots+\frac{1}{j-1}\right)+B_{j}\right\} .
$$

If $j=1$, then

$$
\frac{d}{d s}\left(\begin{array}{c}
-s \\
1
\end{array}\right) Z(s+1)=-B_{1} .
$$

Hence by (7)

$$
-F^{\prime}(0)=-Z^{\prime}(0,-\lambda)+Z^{\prime}(0)+\sum_{j=1}^{h} \frac{B_{j}}{j} \lambda^{j}+\sum_{j=2}^{h} \frac{A_{j}}{j}\left(1+\frac{1}{2}+\cdots+\frac{1}{j-1}\right) \lambda^{j}
$$

and the theorem follows by exponentiating both sides.

The zeta function is easiest to study for a class of sequences that we will call admissible. We will call the sequence $\lambda_{k}$ admissible if (1) the sequence lies in the half-plane $\operatorname{Re} \lambda>0$, (2) the function $\sum_{k=0}^{\infty} e^{-\lambda_{k} t}$ converges absolutely for $t>0$ and has a full asymptotic expansion $\sum_{\nu=0}^{\infty} c_{j_{\nu}} t^{j_{\nu}}, j_{0}<j_{1}<j_{2}<\cdots \rightarrow \infty$, as $t \rightarrow 0$, and (3) $\lim _{t \rightarrow 0} \sum_{k=0}^{\infty}\left|e^{-\lambda_{k} t}\right| t^{\beta}=0$ for some $\beta>0$. The sequences we study below will be rotations of admissable sequences, i.e., of the form $\alpha \lambda_{k}$ for an admissible sequence $\lambda_{k}$. 
With the knowledge of the function $\sum_{k=0}^{\infty} e^{-\lambda_{k} t}$, we can study the analytic continuation of $Z(s)$ for an admissible sequence. We first write $Z(s)$ as a Laplace type integral, or Mellin transform. Write

$$
\Gamma(s)=\int_{0}^{\infty} e^{-t} t^{s-1} d t, \quad \operatorname{Re} s>0,
$$

and make the change of variables $t \rightarrow \lambda t(\lambda \neq 0)$ getting

$$
\Gamma(s)=\lambda^{s} \int_{L} e^{-\lambda t} t^{s-1} d t
$$

where $L$ is the ray from the origin through $1 / \lambda$. If $\operatorname{Re} \lambda>0$, it follows by integrating over a closed contour consisting of a wedge of an annulus, and letting the outer radius go to infinity and the inner radius to zero, that

$$
\Gamma(s)=\lambda^{s} \int_{0}^{\infty} e^{-\lambda t} t^{s-1} d t .
$$

Now for $\operatorname{Re} s>\beta$ we sum over $\lambda=\lambda_{k}$, getting

$$
\sum_{k} \lambda_{k}^{-s}=\frac{1}{\Gamma(s)} \int_{0}^{\infty} \sum_{k} e^{-\lambda_{k} t} t^{s-1} d t
$$

Now $Z(s)$ can be analytically continued using the following:

Theorem 3. Suppose $\phi(t)$ is a complex valued continuous function of a real positive variable $t$, with $|\phi(t)| \leq e^{-b t}$ for some $b>0$ and $t$ large. If $\phi(t)$ has the asymptotic expansion

$$
\phi(t) \sim \sum_{\nu=0}^{\infty} c_{j_{\nu}} t^{j_{\nu}} \quad \text { as } t \rightarrow 0^{+},
$$

$j_{0}<j_{1}<\cdots \rightarrow \infty$, then

$$
F(s)=\int_{0}^{\infty} \phi(t) t^{s-1} d t
$$

converges for $\operatorname{Re} s>-j_{0}$ to an analytic function and can be analytically continued to a function meromorphic in the complex plane with simple poles $-j_{\nu}$ and residues $c_{j_{\nu}}$.

Proof. For $N>0$, let

$$
\phi_{N}(t)=\phi(t)-\sum_{\nu=0}^{N} c_{j_{\nu}} t^{j_{\nu}}
$$

and write

$$
\begin{aligned}
F(s) & =\int_{1}^{\infty} \phi(t) t^{s-1} d t+\int_{0}^{1} \sum_{\nu=0}^{N} c_{j_{\nu}} t^{j_{\nu}+s-1}+\int_{0}^{1} \phi_{N}(t) t^{s-1} d t \\
& =\sum_{\nu=0}^{N} c_{j_{\nu}} \frac{1}{j_{\nu}+s}+A(s)
\end{aligned}
$$

where $A(s)$ is analytic for $\operatorname{Re} s>-j_{N+1}$. This holds for $\operatorname{Re} s>-j_{0}$ but provides an analytic continuation to a function meromorphic in $\operatorname{Re} s>-j_{N+1}$. 
Applying Theorem 3 to $Z(s)$ for an admissible sequence, letting $\phi(t)=$ $\sum_{k} e^{-\lambda_{k} t}$, and $Z(s)=F(s) / \Gamma(s)$, we see that if $j_{\nu} \neq 0,1,2, \ldots$ then $Z(s)$ has a pole at $-j_{\nu}$ with residue $c_{j_{\nu}} / \Gamma\left(-j_{\nu}\right)$. We also see that if $m=0,1,2, \ldots$, then $Z(-m)=c_{m}(-1)^{m} m$ ! where by convention, $c_{m}=0$ if $m \neq j_{\nu}$ for some $\nu$. In particular, $Z(0)=c_{0}$. Similarly, letting $\phi(t)=e^{-a t} \sum_{n} e^{-\lambda_{n} t}$, we get

$$
Z(0, a)=\sum_{m \geq 0} c_{-m} \frac{a^{m}}{m !}
$$

This is very useful in view of property (1).

We have the following theorem [SG, p. 458].

Theorem 4. Let $\theta(t)$ denote a function of the real positive variable $t$ possessing an asymptotic expansion

$$
\theta(t) \sim \sum_{\nu=0}^{\infty} c_{k_{\nu}} t^{k_{\nu}}
$$

as $t \rightarrow 0$, where $-1<k_{0}<k_{1}<\cdots \rightarrow \infty$. Assuming that the Laplace integral

$$
\varphi(a)=\int_{0}^{\infty} e^{-a t} \theta(t) d t
$$

has a half-plane of simple convergence, then $\varphi(a)$ possesses the asymptotic expansion

$$
\varphi(a) \sim \sum_{\nu=0}^{\infty} c_{k_{\nu}} \Gamma\left(k_{\nu}+1\right) a^{-\left(k_{\nu}+1\right)}
$$

as $a \rightarrow \infty$ in the angular sector $|\arg a| \leq \delta<\frac{\pi}{2}$.

We remark that the theorem holds also for complex $k_{v}$ with $-1<\operatorname{Re} k_{0}<$ $\operatorname{Re} k_{1}<\cdots \rightarrow \infty$.

For admissible sequences, Theorems 3 and 4 give us asymptotic expansions for $Z(s, a)$ and $\prod_{z}\left(\lambda_{k}+a\right)$ as $a \rightarrow \infty$, provided we have an asymptotic expansion (8) for $\phi(t)=\sum_{k} e^{\lambda_{k} t}$ as $t \rightarrow 0$. Apply Theorem 4 using $\theta(t)=$ $\phi(t) t^{s-1}$ and $k_{\nu}=j_{\nu}+s-1$ we get for $\operatorname{Re} s>-j_{0}$ the expansion

$$
Z(s, a) \sim \sum_{\nu} \frac{\Gamma\left(j_{\nu}+s\right)}{\Gamma(s)} c_{j_{\nu}} a^{-\left(j_{\nu}+s\right)}
$$

as $a \rightarrow \infty,|\arg a| \leq \delta<\frac{\pi}{2}$. We may use instead $\phi(t)=\left(\theta(t)-\sum_{j_{\nu} \leq 0} c_{j_{\nu}} t^{j_{\nu}}\right) t^{s-1}$ to get for $\operatorname{Re} s \geq 0$

$$
Z(s, a)-\sum_{j_{\nu} \leq 0} \frac{\Gamma\left(j_{\nu}+s\right)}{\Gamma(s)} c_{j_{\nu}} a^{-\left(j_{\nu}+s\right)}=\frac{1}{\Gamma(s)} \int_{0}^{\infty} \phi(t) e^{-a t} d t
$$

where we note that the poles of the left-hand side cancel out for $\operatorname{Re} s \geq 0$. Now differentiating both sides at $s=0$, get

$$
Z^{\prime}(0, a)-\left.\frac{d}{d s} \sum_{j_{\nu} \leq 0} \frac{\Gamma\left(j_{\nu}+s\right)}{\Gamma(s)} c_{j_{\nu}} a^{-\left(j_{\nu}+s\right)}\right|_{s=0}=\int_{0}^{\infty} e^{-a t}\left(\theta(t)-\sum_{j_{\nu} \leq 0} c_{j_{\nu}} t^{j_{\nu}}\right) t^{-1} d t
$$


Applying Theorem 4 we get

$$
\begin{aligned}
-\log \prod_{k}\left(\lambda_{k}+a\right)= & Z^{\prime}(0, a) \sim \sum_{m=0}^{\left[-j_{0}\right]} \frac{(-1)^{m}}{m !} a^{m} c_{-m}\left(1+\cdots+\frac{1}{m}-\log a\right) \\
& +\sum_{j_{\nu} \neq 0,-1,-2, \ldots} \Gamma\left(j_{\nu}\right) a^{-j_{\nu}} c_{j_{\nu}}
\end{aligned}
$$

as $a \rightarrow \infty,|\arg a| \leq \delta<\frac{\pi}{2}$.

\section{EXAMPLES}

\section{Example 1.}

$$
\prod_{n=1}^{\infty} n
$$

The classical formula ior the derivative of the Riemann zeta function at 0 , $\zeta^{\prime}(0)=-\log \sqrt{2 \pi}[\mathrm{SG}$, p. 378] gives

$$
\prod_{n=1}^{\infty} n=\sqrt{2 \pi}
$$

Example 2.

$$
\prod_{n=1}^{\infty} n \tau
$$

We get by (1) and the fact that $\zeta(0)=-\frac{1}{2}$ (which can be deduced from (10)) that

$$
\prod_{n=1}^{\infty} n \tau=\tau^{-1 / 2} \prod_{n=1}^{\infty} n
$$

or

$$
\prod_{n=1}^{\infty} n \tau=\sqrt{2 \pi} \tau^{-1 / 2}
$$

\section{Example 3.}

$$
\prod_{n=1}^{\infty}(n+a)
$$

Since $\operatorname{FP} \zeta(1)=\gamma$, Theorem 2 together with (14) gives

$$
\prod_{n=0}^{\infty}(n+a)=a \sqrt{2 \pi} e^{\gamma a} \prod_{n=1}^{\infty}\left(1+\frac{a}{n}\right) \exp \left(\frac{-a}{n}\right)
$$

hence by the well-known canonical product for the gamma function,

$$
\prod_{n=0}^{\infty}(n+a)=\frac{\sqrt{2 \pi}}{\Gamma(a)} \text {. }
$$

This is essentially the formula for the derivative of the generalized Riemann zeta function at $s=0$ [SG, p. 378]. 
The expansion $\sum_{n=1}^{\infty} e^{-n t}=\left(e^{t}-1\right)^{-1}=t^{-1}-\frac{1}{2}+\sum_{k=1}^{\infty}(-1)^{k-1} B_{k} t^{2 k-1} /(2 k) !$, where $B_{k}, k=1,2, \ldots$, are the Bernoulli numbers, together with (13) give the full Stirling expansion for the gamma function

$$
\log \frac{\Gamma(a+1)}{\sqrt{2 \pi}} \sim\left(a+\frac{1}{2}\right) \log a-a+\sum_{k=1}^{\infty}(-1)^{k-1} \frac{B_{k}}{(2 k)(2 k-1)} a^{-(2 k-1)}
$$

as $a \rightarrow \infty,|\arg a| \leq \delta<\frac{\pi}{2}$.

Example 4.

$$
\prod_{n=1}^{\infty}(n u+a)
$$

By (1), (10), and (16),

$$
\prod_{n=1}^{\infty}(n u+a)=u^{-\frac{1}{2}-\frac{a}{u}} \frac{\sqrt{2 \pi}}{\Gamma\left(1+\frac{a}{u}\right)} .
$$

If $\operatorname{Re} u>0$, we can expand $\sum_{n=1}^{\infty} e^{-n u t}$ as above and using (13), we get (19)

$$
-\log \prod_{n=1}^{\infty}(n u+a) \sim\left(\frac{a}{u}+\frac{1}{2}\right) \log a-\frac{a}{u}+\sum_{k=1}^{\infty}(-1)^{k-1} \frac{B_{k}}{2 k(2 k-1)}\left(\frac{a}{u}\right)^{-(2 k-1)} .
$$

as $a \rightarrow \infty,|\arg a| \leq \delta<\frac{\pi}{2}$. Now using (18), we see that (17) holds with $a$ replaced by $a / u$ with $\operatorname{Re} a>0$ and $\operatorname{Re} u>0$. This shows that (17) holds as $a \rightarrow \infty,|\arg a| \leq \delta<\pi$. Thus we get the full Stirling expansion in its strongest form. This shows the advantage of allowing $\lambda_{n}(=n u)$ to take complex values in this theory.

\section{Example 5.}

$$
\prod_{m, n=1}^{\infty}(m u+n v), \quad \frac{v}{u} \notin(-\infty, 0],-\pi<\arg v-\arg u<\pi .
$$

Let $Z(s)=\sum_{m, n=1}^{\infty}(m u+n v)^{-s}$. Since $Z(0)$ remains unchanged if $u$ and $v$ are replaced by $\alpha u$ and $\alpha v$ respectively $(\alpha \neq 0)$, we may assume for the purpose of computing $Z(0)$ that $\operatorname{Re} u>0$ and $\operatorname{Re} v>0$. Since as $t \rightarrow 0$,

$$
\begin{aligned}
\sum_{m, n=1}^{\infty} e^{-(m u+n v) t} & =\frac{1}{e^{u t}-1} \frac{1}{e^{v t}-1} \\
& =\frac{1}{u v} \frac{1}{t^{2}}-\frac{1}{2}\left(\frac{1}{u}+\frac{1}{v}\right) \frac{1}{t}+\frac{1}{4}+\frac{1}{12}\left(\frac{u}{v}+\frac{v}{u}\right)+O(t)
\end{aligned}
$$

we see by $(10)$ that $Z(0)=\frac{1}{4}+\frac{1}{12}\left(\tau+\frac{1}{\tau}\right)$ where $\tau=v / u$. Thus by (1),

$$
\prod_{m, n=1}^{\infty}(m u+n v)=u^{\frac{1}{4}+\frac{1}{12}\left(\tau+\frac{1}{\tau}\right)} \prod_{m, n=1}^{\infty}(m+n \tau) \text {. }
$$

To compute the latter product, we let $Z(s)=\sum_{m, n=1}^{\infty}(m+n \tau)^{-s}$ and $Z_{1}(s, a)$ $=\sum_{m=1}^{\infty}(m+a)^{-s}$, where we understand these functions to be analytically continued to entire meromorphic functions. Theorem 4 and the methods outlined 
above show that letting $B(s, a)=Z_{1}(s, a)-\frac{1}{(s-1)} a^{1-s}+\frac{1}{2} a^{-s}-\frac{s}{12} a^{-1-s}$, we have $B(s, a)=O\left(|a|^{-3-s}\right)$ for $\operatorname{Re} s>-3$ as $a \rightarrow \infty,|\arg a| \leq \delta<\pi$. Letting $A(s)=Z(s)-\frac{\tau^{1-s}}{s-1} \zeta(s-1)+\frac{1}{2} \tau^{-s} \zeta(s)-\frac{s}{12} \tau^{-1-s} \zeta(s+1)$ we have for $\operatorname{Re} s>-2, A(s)=\sum_{n=1}^{\infty} B(s, n \tau)$. We now differentiate both sides getting $A^{\prime}(0)=\sum_{n=1}^{\infty} B^{\prime}(0, n \tau)$. Using the fact that $\zeta(-1)=\frac{-1}{12}, \zeta(0)=-\frac{1}{2}$, $\zeta^{\prime}(0)=-\frac{1}{2} \log 2 \pi$, and $Z_{1}^{\prime}(0, a)=\log (\sqrt{2 \pi} / \Gamma(1+a))$, we get

$$
\begin{aligned}
-Z^{\prime}(0)= & (\log \tau)\left(\frac{1}{4}+\frac{1}{12}\left(\tau+\frac{1}{\tau}\right)\right)-\frac{\gamma}{12 \tau}-\frac{\tau}{12}-\frac{1}{4} \log 2 \pi+\tau \zeta^{\prime}(-1) \\
& +\sum_{n=1}^{\infty}\left(-\log \Gamma(1+n \tau)+\left(\frac{1}{2}+n \tau\right) \log n \tau-n \tau+\log \sqrt{2 \pi}+\frac{1}{12 n \tau}\right) .
\end{aligned}
$$

Exponentiating both sides of the above equation gives

$$
\begin{gathered}
\prod_{m, n=1}^{\infty}(m+n \tau)=(2 \pi)^{-\frac{1}{4}} \tau^{\frac{1}{4}+\frac{1}{12}\left(\tau+\frac{1}{\tau}\right)} e^{P(\tau)} \prod_{n=1}^{\infty} \Gamma(1+n \tau)^{-1} e^{Q(n \tau)} \\
\text { where } P(\tau)=\frac{-\gamma}{12 \tau}-\frac{\tau}{12}+\tau \zeta^{\prime}(-1) \text { and } \\
Q(x)=\left(\frac{1}{2}+x\right) \log x-x+\log \sqrt{2 \pi}+\frac{1}{12 x}
\end{gathered}
$$

Example 6.

$$
\rho_{2}(u, v)=\prod_{m, n=0}^{\infty}(m u+n v), \quad \frac{v}{u} \notin(-\infty, 0],-\pi<\arg v-\arg u<\pi .
$$

We have by (2) that

$$
\begin{aligned}
\prod_{m, n=0}^{\infty}(m u+n v) & =\prod_{m=1}^{\infty}(m u) \prod_{n=1}^{\infty}(n v) \prod_{m, n=1}^{\infty}(m u+n v) \\
& =2 \pi u^{-\frac{1}{2}} v^{-\frac{1}{2}} \prod_{m, n=1}^{\infty}(m u+n v) .
\end{aligned}
$$

Thus

$$
\prod_{m, n=0}^{\infty}(m u+n v)=2 \pi u^{-\frac{3}{4}+\frac{1}{12}\left(\tau+\frac{1}{\tau}\right)} \tau^{-\frac{1}{2}} \prod_{m, n=1}^{\infty}(m+n \tau)
$$

and the latter product is given by (20). In particular, for $n=1, v=\tau$,

$$
\rho(1, \tau)=(2 \pi)^{\frac{3}{4}} \tau^{-\frac{1}{4}+\frac{1}{12}\left(\tau+\frac{1}{\tau}\right)} e^{P(\tau)} \prod_{n=1}^{\infty} \Gamma(1+n \tau)^{-1} e^{Q(n \tau)}
$$

where $P$ and $Q$ are as in (20). (See [S1, p. 193].)

We remark that $\rho_{2}(u, v)$ is Barnes' double Stirling modular form. It is so called because $\log \rho_{2}(u, v)$ is the constant term in the asymptotic expansion for Barnes' double gamma function, as we will show in the discussion of the next example. 


\section{Example 7.}

$$
\prod_{m, n=0}^{\infty}(z+m u+n v) \quad \text { with } v / u \notin(-\infty, 0]
$$

The function

$$
\Gamma_{2}(z ; u, v)=\rho_{2}(u, v) / \prod_{m, n=0}^{\infty}(z+m u+n v)
$$

is essentially Barnes' double gamma function defined in analogy with (16). By definition $\lim _{z \rightarrow 0} z \Gamma_{2}(z)=1$. The functional equations for $\Gamma_{2}$ are easily derived from (1) and (2). We have

$$
\Gamma_{2}(z+u) / \Gamma_{2}(z)=\prod_{n=0}^{\infty}(z+n v)=v^{\frac{1}{2}-\frac{z}{v}} \prod_{n=0}^{\infty}\left(n+\frac{z}{v}\right)=v^{\frac{1}{2}-\frac{z}{v}} \sqrt{2 \pi} / \Gamma\left(\frac{z}{v}\right) .
$$

Thus we have the functional equation

$$
\Gamma\left(\frac{z}{v}\right) \Gamma_{2}(z+u)=\sqrt{2 \pi} v^{\frac{1}{2}-\frac{z}{v}} \Gamma_{2}(z) .
$$

The same equation holds with $u$ and $v$ interchanged.

We remark that $\Gamma_{2}(z ; 1,1)(2 \pi)^{-z / 2}$ is the double gamma function as referred to in [Va] and [Vi]. The normalizations used here and in those papers are not the same as in Barnes.

Theorem 2 gives the double gamma function in terms of a Weierstrass product. We consider the case when $u=1, v=\tau$. Let $Z(s)=\sum_{m, n=0}^{\prime \infty}(m+n \tau)^{-s}$. Denoting $\Gamma_{2}(z ; 1, \tau)$ by $\Gamma_{2}(z ; \tau)$, we have

$$
\Gamma_{2}(z ; \tau)^{-1}=z e^{Q(z)} \prod_{m, n=0}^{\infty}\left(1+\frac{z}{m+n \tau}\right) e^{P_{2}\left(\frac{-z}{m+n \tau}\right)}
$$

where $Q(z)=F P Z(1) z-(F P Z(2)+\operatorname{Res} Z(2)) \frac{z^{2}}{2}$ and $P_{2}(x)=x+\frac{x^{2}}{2}$.

By use of Theorem 3, we can easily determine that Res $Z(2)=\frac{1}{\tau}$. The numbers $F P Z(1)$ and $F P Z(2)$ are related to Barnes' double modular constants $\gamma_{12}$ and $\gamma_{22}$ [B]. Barnes defined these as constant terms in certain asymptotic expansions. To show the equivalence of the two formulations, we define Barnes' modular constants $C(\tau)$ and $D(\tau)$ by

$$
\begin{aligned}
& \sum_{n=1}^{N} \psi(n \tau)=C(\tau)+\left(N+\frac{1}{2}-\frac{1}{2 \tau}\right) \log N \tau-N+O\left(\frac{1}{N}\right), \\
& \sum_{n=1}^{N} \psi^{\prime}(n \tau)=D(\tau)+\frac{\log N \tau}{\tau}+O\left(\frac{1}{N}\right)
\end{aligned}
$$

as $N \rightarrow \infty$, where $\psi(z)=\frac{d}{d z} \log \Gamma(z)$. The existence of these constants is assured by the Euler-Maclaurin summation formula [B].

It can be shown that

$$
F P Z(1)=-C(\tau)+\gamma, \quad F P Z(2)=D(\tau)+\frac{\pi^{2}}{6}-\frac{1}{\tau}
$$


We will demonstrate only the equation for $F P Z(2)$ in (25), the equation for $F P Z(1)$ being obtained similarly. Let $Z_{1}(s)=\sum_{m, n=1}^{\infty}(m+n \tau)^{-s}$ and $Z_{2}(s, a)=\sum_{m=1}^{\infty}(m+a)^{-s}$. Using Theorem 4 we obtain that $Z_{2}(s, a)-\frac{a^{-s+1}}{s-1}=$ $O\left(|a|^{-s}\right)$ for $s>0$ and $a \rightarrow \infty,|\arg a| \leq \delta<\pi$. Note that the function on the left-hand side is analytic for $\operatorname{Re} s>0$. Now letting $a=n \tau$ and summing we get for $\operatorname{Re} s>0$,

$$
\sum_{n=1}^{\infty}\left(Z_{2}(s, n \tau)-\frac{(n \tau)^{-s+1}}{s-1}\right)=Z_{1}(s)-\frac{\zeta(s-1)}{s-1} \tau^{-s+1} .
$$

Now taking finite parts of both sides of (26) at $s=2$ we get

$$
\sum_{n=1}^{\infty}\left(Z_{2}(2, n \tau)-\frac{1}{n \tau}\right)=F P Z_{1}(2)-\frac{\gamma}{\tau}+\frac{1}{\tau}+\frac{\log \tau}{\tau}
$$

The left-hand side of (27) is evaluated using the fact that

$$
Z_{2}(2, n \tau)=\psi^{\prime}(n \tau)-\frac{1}{(n \tau)^{2}}
$$

and

$$
\sum_{n=1}^{N} \frac{1}{n}=\log N+\gamma+O\left(\frac{1}{N}\right), \quad \sum_{n=1}^{N} \frac{1}{n^{2}}=\frac{\pi^{2}}{6}+O\left(\frac{1}{N}\right)
$$

as $N \rightarrow \infty$. It follows from (27), (28) and (29) that

$$
\sum_{n=1}^{\infty}\left(Z_{2}(2, n \tau)-\frac{1}{n \tau}\right)=D(\tau)-\frac{1}{\tau^{2}} \frac{\pi^{2}}{6}+\frac{\log \tau}{\tau}-\frac{\gamma}{\tau}
$$

Hence by (27) and (30),

$$
F P Z_{1}(2)=D(\tau)-\frac{1}{\tau}-\frac{\pi^{2}}{6 \tau^{2}}
$$

Now $Z(s)=Z_{1}(s)+\zeta(s)\left(1+\tau^{-s}\right)$ and taking finite parts of both sides gives $F P Z(2)$ in (25). To get $F P Z(1)$ in (25), one proceeds similarly using $Z_{2}(s, a)$ $-\frac{a^{-s+1}}{s-1}+\frac{1}{2} a^{-s}=O\left(|a|^{-1-s}\right)$ for $\operatorname{Re} s>-1$.

We now explain the role of $\rho_{2}(u, v)$ in the asymptotic expansion of $\Gamma_{2}(z)=$ $\Gamma_{2}(z ; u, v)$. We have

$$
\sum_{m, n=0}^{\infty} e^{-(m u+n v) t}=\frac{1}{u v} \cdot \frac{1}{t^{2}}+\frac{1}{2}\left(\frac{1}{u}+\frac{1}{v}\right) \cdot \frac{1}{t}-\frac{3}{4}+\frac{1}{12}\left(\frac{u}{v}+\frac{v}{u}\right)+O(t)
$$

as $t \rightarrow 0^{+}$. Hence by (13)

$$
\begin{aligned}
\log \frac{\Gamma_{2}(z)}{\rho_{2}(u, v)} \sim & \frac{1}{2} \frac{1}{u v} z^{2}\left(\frac{3}{2}-\log z\right)-\frac{1}{2}\left(\frac{1}{u}+\frac{1}{v}\right)(1-\log z) \\
& -\left\{-\frac{3}{4}+\frac{1}{12}\left(\frac{u}{v}+\frac{v}{u}\right)\right\} \log z+O\left(\frac{1}{z}\right)
\end{aligned}
$$

as $z \rightarrow \infty$ in any closed sector not containing the closed sector between the vector $-u$ and $-v$. Thus $\log \rho_{2}(u, v)$ is the constant term in the asymptotic expansion of $\log \Gamma_{2}(z)$, analogous to $\log \sqrt{2 \pi}$ in the Stirling expansion for the gamma function. 


\section{Example 8.}

$$
\prod_{n=-\infty}^{\infty}|n+a|^{2}, \quad a=b+c i
$$

By Theorem 2, we have

$$
\prod_{n=-\infty}^{\infty}|n+a|^{2}=\prod_{n=-\infty}^{\infty}(n+b)^{2} \prod_{n=-\infty}^{\infty}\left(1+\frac{c^{2}}{(n+b)^{2}}\right)
$$

We note that for $\lambda_{k}$ real $\left(\arg \lambda_{k}=0\right)$,

$$
\prod_{k}\left(\lambda_{k}+a\right)^{2}=\left(\prod_{k}\left(\lambda_{k}+a\right)\right)^{2} \text {. }
$$

Now by (16) and the identity $\Gamma(z) \Gamma(1-z)=\pi \csc \pi z$ we get

$$
\prod_{n=-\infty}^{\infty}(n+b)^{2}=4 \sin ^{2} \pi b
$$

Thus by (33) and (34),

$$
\begin{aligned}
\prod_{n=-\infty}^{\infty}|n+a|^{2} & =4 b^{2} \prod_{n=-\infty}^{\infty}\left(1+\frac{b}{n}\right)^{2} \prod_{n=-\infty}^{\infty}\left(1+\frac{c^{2}}{(n+b)^{2}}\right) \\
& =4 \pi^{2}|a|^{2} \prod_{n=-\infty}^{\prime}\left|1+\frac{a}{n}\right|^{2} .
\end{aligned}
$$

Hence

$$
\prod_{n=-\infty}^{\infty}|n+a|^{2}=4|\sin \pi a|^{2}
$$

Example 9.

$$
\prod_{m, n=-\infty}^{\infty}|m+n \tau|^{2}, \quad \operatorname{Im} \tau>0
$$

The evaluation of this product is Kronecker's limit formula,

$$
\prod_{m, n=-\infty}^{\infty}|m+n \tau|^{2}=(2 \pi)^{2}|\eta(\tau)|^{4}
$$

where $\eta$ is Dedekind's eta function $\eta(\tau)=e^{\pi i \tau / 12} \prod_{k}^{\infty}\left(1-e^{2 \pi i k \tau}\right), \operatorname{Im} \tau>0$.

Using the theory above, we are able to give a new derivation of (36). Let $Z(s)=\sum_{n=1}^{\infty} \sum_{m=-\infty}^{\infty}\left(|m+n \tau|^{2}\right)^{-s}$, and $Z_{1}(s, c)=\sum_{m=-\infty}^{\infty}\left(|m+a|^{2}\right)^{-s}$ where $a=b+i c, b$ fixed. Using Theorem 4 , we can get an asymptotic expansion for $Z_{1}$, as $c \rightarrow \infty$ if we have an expansion for $\sum_{m=-\infty}^{\infty} e^{-(m+b)^{2} t}$ as $t \rightarrow 0^{+}$. The corresponding integral is

$$
\int_{-\infty}^{\infty} e^{-(x+b)^{2} t} d x=\sqrt{\pi} t^{-\frac{1}{2}}
$$


and as $t \rightarrow 0$, the difference between the sum and the integral can easily be shown to be $O\left(t^{n}\right)$ for all $n>0$. This also follows from Jacobi's identity [DM, p. 52]. Thus,

$$
\sum_{m=-\infty}^{\infty} e^{-(m+b)^{2} t} \sim \sqrt{\pi} t^{-\frac{1}{2}}
$$

as $t \rightarrow 0^{+}$is a full asymptotic expansion. Now Theorem 4 and (37) give that

$$
Z_{1}(s, c)-\sqrt{\pi} \frac{\Gamma\left(-\frac{1}{2}+s\right)}{\Gamma(s)} c^{-2 s+1}=O\left(c^{-2 s-2}\right)
$$

as $c \rightarrow \infty$ for $\operatorname{Re} s>-1$.

Letting $a=n(x+i y)$, equation (39) gives upon summation over $n$

$$
\begin{gathered}
\sum_{n=1}^{\infty}\left(Z_{1}\left(s, n^{2} y^{2}\right)-\sqrt{\pi} \frac{\Gamma\left(-\frac{1}{2}+s\right)}{\Gamma(s)}(n y)^{-2 s+1}\right) \\
=Z(s)-\sqrt{\pi} \frac{\Gamma\left(-\frac{1}{2}+s\right)}{\Gamma(s)} \zeta(2 s-1) y^{1-2 s}
\end{gathered}
$$

for $\operatorname{Re} s>-1$. Now differentiating (39) at $s=0$ and using $(34), \zeta(-1)=-\frac{1}{12}$, and $\Gamma\left(-\frac{1}{2}\right)=-2 \sqrt{\pi}$ we get

$$
\sum_{n=1}^{\infty}\left(-\log 4|\sin n \pi \tau|^{2}+2 \pi n y\right)=Z^{\prime}(0)-\frac{\pi y}{6}
$$

Exponentiating both sides gives

$$
\prod_{\substack{m=-\infty \\ n=1}}^{\infty}|m+n \tau|^{2}=|\eta(\tau)|^{2}
$$

Now (36) easily follows from (1) since

$$
\prod_{m=-\infty}^{\infty} m^{2}=(2 \pi)^{2}
$$

Example 10.

$$
\prod_{m=-\infty}^{\infty}(m+a), \quad \operatorname{Im} a \neq 0,-\pi<\arg (m+a)<\pi .
$$

If $\operatorname{Im} a>0$, we have $-(m+a)=e^{\pi i}(m+a)$, hence

$$
\begin{aligned}
\prod_{m=-\infty}^{\infty}(m+a) & =a \prod_{m=1}^{\infty}(m+a) \prod_{m=1}^{\infty} e^{\pi i}(m-a) \\
& =a^{-1} \frac{\sqrt{2 \pi}}{\Gamma(a)} e^{\pi i\left(-\frac{1}{2}+a\right)} \frac{\sqrt{2 \pi}}{\Gamma(-a)}=1-e^{2 \pi i a}
\end{aligned}
$$

where we have used (1), (10), (16) and the identity $\Gamma(a) \Gamma(1-a)=\pi / \sin \pi a$. We get a similar result for $\operatorname{Im} a<0$. Summarizing,

$$
\prod_{m=-\infty}^{\infty}(m+a)= \begin{cases}1-e^{2 \pi i a}, & \operatorname{Im} a>0, \\ 1-e^{-2 \pi i a}, & \operatorname{Im} a<0 .\end{cases}
$$




\section{Example 11.}

$$
\prod_{m, n=-\infty}^{\infty}(m+n \tau), \quad \operatorname{Im} \tau>0,-\pi \leq \arg (m+n \tau)<\pi .
$$

Let $Z_{1}(s, a)$ denote the analytic continuation of $\sum_{m=1}^{\infty}(m+a)^{-s}$. We have seen from Theorem 4 that

$$
Z_{1}(s, a)=\frac{a^{1-s}}{s-1}-\frac{1}{2} a^{-s}+\frac{s}{12} a^{-1-s}+O\left(|a|^{-3-s}\right)
$$

for $\operatorname{Re} s>-3, a \rightarrow \infty,|\arg a|<\pi-\delta$. Likewise $Z_{2}(s, a)=e^{\pi i s} Z_{1}\left(s, e^{\pi i} a\right)$ is the analytic continuation of $\sum_{m=1}^{\infty}(-m+a)^{-s}$ where $\arg (-m)=-\pi i$, and substituting in the above, we get

$$
Z_{2}(s, a)=-\frac{a^{1-s}}{s-1}-\frac{1}{2} a^{-s}-\frac{s}{12} a^{-1-s}+O\left(|a|^{-3-s}\right) \quad \text { as } a \rightarrow \infty
$$

as above. Letting $Z(s, a)$ be the analytic continuation of $\sum_{m=-\infty}^{\infty}(m+a)^{-s}$ we have

$$
Z(s, a)=Z_{1}(s, a)+a^{-s}+Z_{2}(s, a)
$$

and adding the two above asymptotic expansions, we have

$$
Z(s, a)=O\left(|a|^{-3-s}\right)
$$

for $\operatorname{Re} s>-3$, as $a \rightarrow \infty,-\pi+\delta<\arg a<-\delta$ or $\delta<\arg a<\pi-\delta$. Checking the residues, we see that the function (43) in an entire function of $s$. Also by (43),

$$
\sum_{n \neq 0}(m+n \tau)^{-s}=\sum_{n=-\infty}^{\infty} Z(s, n \tau)
$$

and the sum over $n$ on the right converges absolutely for $\operatorname{Re} s>-2$, and the analytic continuation of (44) is also an entire function of $s$. Now (44) and the remark following imply that we can take the iterated product:

$$
\begin{aligned}
\prod_{n \neq 0}(m+n \tau) & =\prod_{n=-\infty}^{\infty} \prod_{m=-\infty}^{\infty}(m+n \tau) \\
& =\prod_{n=1}^{\infty} \prod_{m=-\infty}^{\infty}(m+n \tau) \prod_{n=1}^{\infty} \prod_{m=-\infty}^{\infty}(m-n \tau) \\
& =\prod_{n=1}^{\infty}\left(1-e^{2 \pi i n \tau}\right)^{2}=\eta^{2}(\tau) e^{\frac{-\pi i \tau}{6}}
\end{aligned}
$$

where we have used Example 10. Now

$$
\prod_{m=-\infty}^{\infty} m=\pi i
$$

using (1) and Example 1. Hence finally for $\operatorname{Im} \tau>0$,

$$
\prod_{m, n=-\infty}^{\infty}(m+n \tau)=2 \pi i \eta^{2}(\tau) e^{-\frac{\pi i \tau}{6}}=2 \pi i \prod_{n=1}^{\infty}\left(1-e^{2 \pi i n \tau}\right)^{2}
$$


Comparing (45) and (41) or (42) and (35) we see that in general, $\Pi \lambda_{k} \prod \bar{\lambda}_{k} \neq$ $\Pi\left|\lambda_{k}\right|^{2}$.

We could also demonstrate (45) by writing the product on the left in terms of the product of 4 products of the type in Example 6 and using the identity $\rho_{2}(1, \tau) \rho_{2}(1,-\tau)=(2 \pi)^{3 / 2} \tau^{-1 / 2} \eta(\tau) \exp \left((\pi i / 12)\left(3+\frac{1}{\tau}\right)\right)$.

\section{Example 12.}

$$
\begin{array}{ll}
\prod_{m, n=-\infty}^{\infty}(m+n \tau), & \operatorname{Im} \tau>0, \arg \tau-2 \pi \leq \arg (m+n \tau)<\arg \tau, \\
& 0<\arg \tau<\pi .
\end{array}
$$

This example, when compared with Example 11, illustrates the dependence of the zeta regualrized product on the argument of the factors. Let $P_{2}(\tau)$ be the product with the argument chosen as in Example 12 and $P_{1}(\tau)$ with the argument chosen as in Example 11. We have by (2),

$$
P_{2}(\tau)=\frac{P_{1}(\tau) \prod_{\substack{m=0 \\ n=1}}^{\infty} e^{-2 \pi i}(-m+n \tau)}{\prod_{\substack{m=0 \\ n=1}}^{\infty}(-m+n \tau)} .
$$

Letting $Z(s)=\sum_{m=0 ; n=1}^{\infty}(-m+n \tau)^{-s}$ we compute using computations in Example 5 that $Z(0)=-\frac{1}{4}-\frac{1}{12}\left(\tau+\frac{1}{\tau}\right)$. Now using (2) and (46), we get

$$
P_{2}(\tau)=i P_{1}(\tau) e^{\frac{\pi i}{6}\left(\tau+\frac{1}{\tau}\right)}
$$

Also by (1) we get

$$
P_{1}\left(-\frac{1}{\tau}\right)=(-\tau) P_{2}(\tau)
$$

Equations (47),(45) and (48) easily imply the functional equation $\eta^{2}(-1 / \tau)=$ $-i \tau \eta^{2}(\tau)$ for the eta function, giving another approach to this identity using zeta regularized products. For other proofs, see [A, Chapter 3].

Example 13.

$$
\prod_{m, n=-\infty}^{\infty}(m+n \tau+z), \quad \operatorname{Im} \tau>0,-\pi \leq \arg (m+n \tau)<\pi .
$$

Let $Z(s)=\sum_{\gamma \in \Omega} \gamma^{-s}$ where $\Omega$ is the lattice with basis $1, \tau$ and $-\pi \leq$ $\arg \gamma<\pi$. We have

$$
Z(s)=\zeta(s)+\left(e^{-\pi i}\right)^{-s} \zeta(s)+\sum_{n \neq 0}(m+n \tau)^{-s}
$$

The latter sum can be converted to an iterated sum as in (44). Since $Z$ is an entire function of $s$, Theorem 2 gives

$$
\prod_{\gamma \in \Omega}(\gamma+z)=\left(\prod_{\gamma \in \Omega}^{\prime} \gamma\right) \exp \left(-\frac{Z(2)}{2} z^{2}+Z(1) z\right) \sigma(z)
$$


where $\sigma$ is the Weierstrass product associated with the sequence $\gamma$, i.e., the Weierstrass sigma function.

To compute $Z(2)$ and $Z(1)$, note that by (49) and (44) we can compute these values by iterated summation. For $Z(2)$ this is just Eisenstein summation, which is equivalent to analytic continuation. By the well-known Eisenstein summation formula [A, p. 71, problem 5]

$$
Z(2)=-4 \pi i \frac{\eta^{\prime}(\tau)}{\eta(\tau)}
$$

We may compute directly from (49) that

$$
Z(1)=-\pi i, \quad Z(0)=-1 .
$$

(For a discussion of the relationship between Eisenstein summation and analytic continuation, see [W].)

Hence, finally, using (49), (50), (51) and Example 11,

$$
\prod_{z}(\gamma+z)=2 \pi i \eta^{2}(\tau) \exp \left(-\frac{\pi i \tau}{6}+2 \pi i \frac{\eta^{\prime}(\tau)}{\eta(\tau)} z^{2}-\pi i z\right) \sigma(z)
$$

If $\vartheta_{1}(z)$ in Jacobi's theta function as in [SG] we can derive the relationship

$$
\sigma(z)=\vartheta_{1}(z)(2 \pi)^{-1} \eta^{-3}(\tau) \exp \left(-2 \pi i \frac{\eta^{\prime}(\tau)}{\eta(\tau)} z^{2}\right)
$$

Hence the theta function is given as a zeta regularized product by

$$
\prod_{z}(\gamma+z)=i \eta^{-1}(\tau) \exp \left(-\frac{\pi i \tau}{6}-\pi i z\right) \vartheta_{1}(z)
$$

We note that the functional equation for (53) can be written

$$
\prod_{z}(\gamma+(z+\tau))=-e^{-2 \pi i(z+\tau)} \prod_{z}(\gamma+z) .
$$

This can also be derived from property (1) of the zeta regularized product with an argument similar to the one in Example 12. Care must be taken to distinguish between $\prod_{z}(\gamma+(z+\tau))$ and $\prod_{z}((\gamma+\tau)+z)$ as indicated by the convention before Theorem 1 .

Equation (45) can be used to find the product of the nonzero eigenvalues of $\partial / \partial \bar{z}$ on a flat torus. If the torus is $\mathbb{C} / \Lambda$, where $\Lambda$ is the lattice generated by 1 and $\tau, \operatorname{Im} \tau>0$, then the eigenvalues of $\partial / \partial \bar{z}$ are $\pi i \gamma^{*}$ for $\gamma^{*}$ in the dual lattice $\Omega^{*}$ generated by $i(\operatorname{Im} \tau)^{-1}$ and $i \tau(\operatorname{Im} \tau)^{-1}$. Likewise the eigenvalues of $\partial / \partial z$ are $\pi i \bar{\gamma}^{*}$ and of $(\partial / \partial z)(\partial / \partial \bar{z})=\frac{1}{4} \Delta$ are $\pi^{2}\left|\gamma^{*}\right|^{2}$. If we let $\operatorname{det}^{\prime} \partial / \partial \bar{z}$ denote the zeta regularized product of the nonzero eigenvalues, then

$$
\operatorname{det}^{\prime} \frac{\partial}{\partial \bar{z}}=\prod_{\gamma \in \Omega}^{\prime}(-\pi)(\operatorname{Im} \tau)^{-1} \gamma
$$

If we use the principal value of arg as in Example 11, then using (1) and (45) we get

$$
\operatorname{det}^{\prime} \frac{\partial}{\partial \bar{z}}=-2 i(\operatorname{Im} \tau) \eta^{2}(\tau) e^{-\frac{\pi i \tau}{6}}
$$

Similarly, $\operatorname{det} \partial / \partial z$ is the conjugate of (54). We note that by (41),

$$
\operatorname{det}^{\prime} \frac{\partial}{\partial z} \frac{\partial}{\partial \bar{z}}=4|\operatorname{Im} \tau|^{2}|\eta(\tau)|^{4}
$$


hence (54), (55) and (56) show that

$$
\operatorname{det}^{\prime} \frac{\partial}{\partial z} \operatorname{det}^{\prime} \frac{\partial}{\partial \bar{z}} \neq \operatorname{det}^{\prime} \frac{\partial}{\partial z} \frac{\partial}{\partial \bar{z}} \text {. }
$$

The following additional references containing related results on zeta regularized products have come to our attention since this paper was written. Compare with [DP, p. 1002].

J. Jorgenson and S. Lang, Some analytic properties of regularized products, preprint.

C. Deninger, Local L-factors of motives and regularized products, Invent. Math. 107 (1992), 135-150.

\section{ACKNOWLEDGMENTS}

We would like to thank J. Choi and M. Hughes for their help in preparing this paper.

\section{REFERENCES}

[A] T. Apostol, Modular functions and Dirichlet series in number theory (2nd ed.), SpringerVerlag, New York, 1990.

[B1] E. W. Barnes, Genesis of the double gamma function, Proc. London Math. Soc. 31 (0000), 358-381.

[B2] _ The theory of the double gamma function, Philos. Trans. Royal Soc. (A) 196 (1901), 265-388.

[B3] _ The theory of the multiple gamma function, Trans. Cambridge Philos. Soc. 19 (1904), 374-425.

[CQ] J. Choi and J. R. Quine, Zeta regularized products and functional determinants on spheres, preprint.

[DP] E. D'Hoker and D. H. Phong, The geometry of string perturbation theory, Rev. Mod. Phys. 60 (1988), 917-1065.

[DM] H. Dym and H. P. McKean, Fourier series and integrals, Academic Presss, 1972.

[G] P. Gilkey, Invariance theory, the heat equation and the Atiyah-Singer index theorem, Publish or Perish, Wilmington, Del., 1984.

[MS] H. P. McKean and I. M. Singer, Curvature and the eigenvalues of the Laplacian, J. Differential Geom. 1 (1967), 43-69.

[O] T. Orloff, Another proof of the Kronecker limit formula, Number Theory, (H. Kisilevsky and J. Labute, Eds.), Amer. Math. Soc., Providence, R.I., 1987.

[OPS] B. Osgood, R. Phillips, and P. Sarnak, Extremals of determinants of Laplacians, J. Funct. Anal. 80 (1988), 148-211.

[QS] J. R. Quine and R. Y. Song, A double Stirling asymptotic formula, preprint.

[RS] D. B. Ray and I. M. Singer, R-torsion and the Laplacian on Riemannian manifolds, Adv. in Math. 7 (1974), 145-210.

[SG] G. Sansone and J. Gerretsen, Lectures on the theory of functions of a complex variable, Noordhoff, Groningen, 1960.

[S1] T. Shintani, A proof of the classical Kronecker limit formula, Tokyo J. Math. 3 (1980), 191-199.

[S2] _ On a Kronecker limit formula for real quadratic fields, J. Fac. Sci. Univ. Tokyo Sect. IA Math., 24 (1977), 167-199.

[Va] I. Vardi, Determinants of Laplacians and multiple gamma functions, SIAM J. Math. Anal. 19 (1988), 493-507. 
[Vi] M. F. Vignéras, L'équation fonctionelle de la fonction zeta de Selberg du groupe modulaire $\operatorname{PSL}(2, Z)$, Astérisque 61 (1979), 235-249.

[Vo] A. Voros, Spectral functions, special functions and the Selberg zeta function, Comm. Math. Phys. 110 (1987), 439-465.

[W] A. Weil, Elliptic functions according to Eisenstein and Kronecker, Springer-Verlag, New York, 1976.

Department of Mathematics, The Florida State University, Tallahassee, Florida 32306-3027

E-mail address, J. R. Quine: quine@math.fsu.edu

E-mail address, R. Y. Song: song@math.fsu.edu 